\title{
On Core Concepts and Terminology
}

\author{
Anna Maria Di Sciullo, reply by Evelina Leivada
}

In response to "Misused Terms in Linguistics" (Vol. 5, No. 2).

To the editors:

In her essay, Evelina Leivada reports on a range of terminology issues in linguistics. These are drawn from a variety of sources, including citations from articles, blogs, talks, and interactions at conferences. Linguists are urged to follow the lead of psychologists and discuss "lists of inaccurate, ambiguous, misused, and polysemous terms." In closing, Leivada states that clarity matters in linguistics and has consequences for the visibility of linguistics in neighboring fields.

While it is difficult not to agree with this general conclusion, it is also difficult not to view terminology issues, namely problems with labels for concepts, as an epiphenomenon of the rapid evolution of linguistics and the different approaches to language pursued within the generative enterprise, in cognitive sciences and beyond. Although terminology issues are raised at the forefront, the crux of the matter with this essay is not terminology per se, but different views of core concepts in linguistics.

The generative enterprise explores a specific approach to language. It is concerned with what has been termed the basic property of language, namely, the property of the mind to construct an infinite array of structured expressions, each one with a semantic interpretation that expresses a thought that can be externalized in one of the sensorimotor systems. In this approach, the language faculty is a generative system that feeds semantic interpretation directly and sensorimotor interpretation only indirectly. ${ }^{1}$

Within the generative enterprise, the biolinguistic program is concerned with language internal to the individual, the I-language, which is distinct from the external language, the E-language. It aims to provide an explanation for I-language by understanding through its biological basis. ${ }^{2}$ Abstracting away from Darwin and the modern synthesis, the biolinguistic program brings to the fore arguments in favor of language as a human-specific trait and the rapid evolution, or emergence, of language. ${ }^{3}$ The ultimate goal of the generative enterprise and the biolinguistic program is to provide a genuine explanation of language that will meet the criteria of learnability. The system needs to be acquired by the individual, as well as the criteria of evolvability, as the innate system of the faculty of language needs to evolve. Such explanation cannot be reached with descriptive or behaviorist approaches to language. ${ }^{4}$

Explanation and simplicity are intrinsically related in this framework. This can be seen in the development of generative grammar. ${ }^{5}$ Current research aims to explain I-language in terms of the first and the third factor in language design. The first factor is the genetic endowment and the third factor is the principles of efficient computation external to the language faculty. ${ }^{6}$

Other approaches based on different or partially different perspectives on language, with partially different arrays of concepts, are available. Terminological issues may arise within linguistics as well as in neighboring fields.

\section{Terminology Issues}

To address the issues in relation to terminology discussed by Leivada, it is useful to differentiate linguistic terms from their use at different points in the development of the generative enterprise. It is also helpful to consider the use of linguistic terms in neighboring sciences. Neuroscience is an interesting case as it faces the map and mapping problem, as pointed out by David Poeppel.7 Nonetheless, it has been established that Broca's area, corresponding to BA44/45 in the frontal lobe of the brain's dominant hemisphere, supports the processing of syntax in general. ${ }^{8}$ A subdivision of syntactic computations within Broca's area for complex syntactic structures has been demonstrated with BA44 activated for center-embedding and for sentences involving displacement of syntactic constituents, and BA45 selectively adapted to displacement. ${ }^{9}$ Psycholinguistics is also interesting as it faces the problem of attesting the psychological reality of linguistic concepts. It is difficult to probe the abstract properties of I-language with behavioral experiments alone, and brain imaging studies are often used in addition to behavioral experiments. The best possible outcome is when results 
from independent studies converge. Biolinguistics is perhaps the most interesting case as it relies on interactions between different sciences including linguistics, biology, mathematics, and physics.

There is no doubt that clarity and coherence are imperative in any field of inquiry, whether theoretical or applied, disciplinary or interdisciplinary. To this end, it is instructive to reflect on how terms for core notions in a given field emerge, evolve, disappear, and also sometimes reappear as relics from past eras to measure achievements and identify new problems. The misuse of terms in linguistics might be better understood by reflecting on the life of core concepts in the field and on the terms used to investigate them. It may also be helpful to consider terms used to study core concepts in linguistics as names for objects yet to be better understood. The terms used for given concepts will change over time; some will be redefined or eliminated in light of advances made in their understanding and new problems that arise in the investigation.

\section{Terminological Confusion}

Advances have been made through the development of the generative enterprise. During the late 1970s and early 1980 s, the principles and parameters model of grammar replaced the so-called standard theory. ${ }^{10}$ In this model, a grammar of a language is a theory of that language, and Universal Grammar is a theory of all languages. Leivada observes that the terms Universal Grammar (UG) and language universals are misused in linguistics.

UG is sometimes identified with linguistic universals; but this is a mistake. When Chomsky talks about language universals, he does not refer to properties that are universally attested to in all languages, but to computational properties of the mind that are universal because they arise from a species-universal innate ability.

Why would these terms be misused? One possible reason is that they were proposed within different theories of language. As defined by Joseph Greenberg, language universals are not part of Universal Grammar and mainly state universals, absolute and relative, based on the surface distribution of major syntactic constituents: subject, verb, and object. ${ }^{11}$ Not all possible orders are attested in the languages of the world, which indicates that more abstract properties of languages are at play for characterizing Universal Grammar. Greenberg's work is important and enabled the development of further research capable of deriving specific language universals from independent syntactic properties. ${ }^{12}$ This is a step forward in simplifying linguistic theory. Leivada writes:

There is no reason to assume that linguistic universals, understood as properties that are shared across languages, are necessarily derived from UG. Although most languages settle on a consistent word order, this preference does not reflect the imperatives of UG. ... Given that a complete list of all the UG principles has not been compiled, the possibility that these principles are, for the most part, not language-specific, but have cognitive, third factor roots, cannot be ruled out.

Third factor principles are at play in the derivation of syntactic constituents. It has been observed through the diachronic development of the Indo-European languages that languages tend to regularize adpositions to either prepositions or postpositions. Both preposition and postpositions are observed in Latin nominal constituents including a preposition and a personal pronoun, e.g., cum me, me cum. In modern Italian, only the prepositional variant remains, e.g., con me, notwithstanding the fact that more complex structures emerged in old Italian, e.g., con me meco, come, comeco, con esso meco. This historical development has not been attributed to principles of UG, but to third factor principles reducing complexity akin to natural laws. ${ }^{13}$

In current minimalist research, the externalization of linguistic constituents and their absence in some cases has also been attributed to third factor principles of efficient computation external to the basic property of language. This is the case for principles of pronunciation. According to pronounce the minimum, the copy left by a displaced constituent is generally not pronounced, e.g., what did you say? vs. * what did you say what ${ }^{\text {?4 }}$ Other examples in which principles of pronunciation play a role in the derivation of linguistic expressions occur when certain categories, which are not copies of displaced constituents, are not externalized $(<>)$. This is the case for prepositions such as "at" in locative expressions-e.g., I prefer to stay $\langle A T\rangle$ home-discussed by Christopher Collins and elsewhere, as well as unpronounced coordinators in multiple conjunctions-e.g., I saw Paul $<A N D>$ John $<A N D>$ Mary-and several other categories..$^{15}$ That third factor principles are at play in language is not only a possibility as mentioned by Leivada. It is a working hypothesis that has already been proposed and investigated in the generative enterprise for quite some time.

If confusion with the content of linguistic terms arises, whether in linguistics or neighboring fields, it is important to keep in mind that these terms emerge from different approaches. Language universals gave rise to empirical generalizations, some of which have been derived from independent principles in later stages of the generative enterprise. It is important to clarify why language universals cannot be equated with Universal Grammar, why it matters for the understanding of I-language, and why surface phenomena such as word order are subject to principles external to I-language. Such a perspective is absent from Leivada's essay. 


\section{Terminological Fluidity}

Leivada asserts that a degree of terminological fluidity is associated with certain linguistic terms, such as parameters and features. In particular, she draws attention to metaphors of language development used in Chomsky's earlier works, including the notion of instantaneous acquisition. $^{16}$

The problem is that the metaphor of instantaneous acquisition assumes that innateness and the environment are fully separable.... The metaphors of language development were once useful. It is through their subsequent use that they became unhelpful.

On this point, I disagree with Leivada. The notion of parameters, initially defined as options left open in the principles of UG, gave rise to lively research. ${ }^{17}$ The term has been defined in different ways through the development of the generative enterprise, given extended work on linguistic variation, both within single languages and cross-linguistically. Several parameters have been proposed, as well as different formalizations of this notion. Mark Baker's polysynthetic parameter, for example, identifies hierarchical dependencies between parameters. ${ }^{18}$ In the minimalist program, it has been proposed that parameters could be reduced to minimal differences in the features of functional categories. Several parameters have been eliminated and their effects attributed to independent principles of language. These include the head-directionality parameter, which has been proposed to derive from the directionality of parsing. ${ }^{19}$ In his recent book, Ian Roberts redefines parameters in terms of structured hierarchies of features related to third factor principles. ${ }^{20}$ This enables elegant analyses of different historical language changes, including negation. The reduction of parameters to principles external to the language faculty contributes to an explanatory theory of language.

The purpose of the generative enterprise is to provide a genuine explanation for I-language. It comes as no surprise that current minimalist research eliminates the notion of parameters as options left open by UG, as defined in the principles and parameters model, where the different modules of UG were each associated with a set of primitives, axioms, and parameters. ${ }^{21}$

It is unfortunate that Leivada's discussion of the term does not report its current status, as subsumed under third factor principles, and the progress achieved since it was proposed in the principles and parameters model. Instead, her essay focuses on criticisms of the usage of this term during previous stages of the generative enterprise.

Terminological fluidity is not without consequences. In the case of the term "parameter," its misuse over the years raised serious concerns about the biological plausibility of an innate endowment for language that consists of millions of minimal points of variation. "If the number of parameters," Frederick Newmeyer observed, "needed to handle the different grammars of the world's languages, dialects, and (possibly) idiolects is in the thousands (or, worse, millions), then ascribing them to an innate UG to my mind loses all semblance of plausibility."

When confusion arises about the putative terminological fluidity of parameters, it is useful to clarify the notion of parameter and identify its trajectory within the generative enterprise. It is also useful to explain why the notion of parameters is dispensed with in recent works in the minimalist program, along with the different principles of UG they depend upon in the principles and parameters model.

The elimination of the notion of parameter provides an explanation for the absence of acquisition. If language is not learned through experience, that is, by contact with the environment, then the logical problem of language acquisition can be solved. Namely, notwithstanding the fact that the empirical data available to the infant is partial, language acquisition unfolds in a very limited time. ${ }^{22}$ The optimal hypothesis is that no learning takes place.

Leivada's discussion of the term feature also reports criticisms instead of reporting the progress made from the introduction of syntactic features in the standard theory to current understanding in the minimalist program. The hypothesis that syntactic features are part of the syntactic component of the grammar was proposed by Chomsky as part of the standard theory. ${ }^{23}$ He extended this idea in "Remarks on Nominalizations," where nouns, verbs, adjectives, and prepositions are defined in terms of two binary features $[ \pm \mathrm{N}]$ and $[ \pm \mathrm{V}] .{ }^{24}$ The introduction of syntactic features enabled cross-categorial generalizations and provided an account for natural classes of categories. ${ }^{25}$ In the minimalist framework, syntactic features associated with syntactic constituents enter into local agreement relations, which may lead to displacement. ${ }^{26}$ Features have been proposed to account for the fact that syntactic constituents are displaced to higher positions in syntactic structures. Certain syntactic features, such as the extended projection principle feature mentioned by Leivada, have been eliminated. Their effects are derived by independent properties, including labeling and simplest, ${ }^{27}$ according to which Merge, the core combinatorial operation of the language faculty, applies freely. Derivations are canceled if, for example, constituents cannot be labeled.

While Norbert Hornstein is right in suggesting that "the real problem is that we have no hint of a theory of features," as quoted by Leivada, support for a limited set of syntactic features in linguistic theory comes from the fact that they feed the semantic interface. The features $[ \pm$ $\mathrm{N}]$ and $[ \pm \mathrm{V}]$ can be thought of as being legible as $[ \pm$ argumental] and [ \pm predicative] at the semantic interface, but not at the sensorimotor interface. This satisfies the strong 
minimalist thesis, according to which language is the best solution to interface legibility conditions.

In the generative enterprise's current state of development, Leivada's discussion on misused terms such as parameters and features is obsolete. If confusion arises with respect to certain terms in linguistics, it is useful to understand why such terms have been proposed, what they accounted for, how their content and role have been modified to provide a genuine explanation for language, and what problems and questions they left open for further research. It goes without saying that criticism is useful to the extent that it leads to alternatives with greater explanatory capacities.

\section{Further Terminological Confusion}

Questions arise among linguists whether the architecture of the language faculty, as defined by Marc Hauser, Chomsky, and W. Tecumseh Fitch, is the optimal solution to the problem of linking the sensorimotor to the conceptual-intentional systems. ${ }^{28}$ "It is harder to argue that all linguistic theories are optimal," Leivada writes, "especially in relation to the preceding suggestions that innateness consists of an unknown, ever-growing number of features, parameters, and other primitives."

This remark is obsolete in relation to current research in the generative enterprise, where features, parameters, and other primitives are reduced to their minimum or subsumed in third factor principles as discussed above. She reports confusion that arose with the term language faculty in the narrow sense (FLN), which Hauser, Chomsky, and Fitch distinguished from the language faculty in the broad sense (FLB):

But, of course, FLN is not the same thing as UG. FLN is unique to humans and unique to language. UG does not have this character. UG is relevant to both FLN's language-specific properties, and FLB's non-language-specific properties. Clearly, FLN is not tantamount to UG. Any suggestions to the contrary represent a misuse of two important terms in linguistics. It is a mistake that many linguists have made.

Linguistics cannot be reduced to terminology. Over time, terms used for given concepts will change; some of them will be redefined or eliminated, given the advances made in understanding and the new problems that arise in the investigation of language.

Chomsky reiterates the particular approach to language pursued in the generative enterprise, which aims to explain the basic property of language. ${ }^{29}$ In this particular approach, a genuine explanation meets two conditions: learnability and evolvability. Given that I-language is internal to the individual, it is not acquired. The simplest explanation for the basic property of language is that it can be explained on the basis of two of the three factors of language design: genetic endowment and the principles of efficient computation. In this perspective, FLN reduces to Merge, the simplest form of which would be internal Merge. An argument to this effect comes from the fact that internal Merge is simpler than external Merge. The latter requires an additional operation of search in the lexicon. Another argument is that internal Merge also derives the successor function in arithmetic. This leads to further problems and questions, including whether there are other variants of Merge, and why external Merge would be necessary.

Leivada's essay is not applicable to current research in the generative enterprise. It is less informative about the misused terms themselves than it is about different approaches to language. The terminological issues discussed in the essay should be understood as differences in the understanding of core concepts in linguistics.

The visibility of linguistics in neighboring fields is important. On this point, I agree with Leivada. But it is surprising that the investigation of core concepts in linguistics would be reduced to normative terminological issues in her essay. The consequences of doing so are indeed detrimental to both linguistics and fruitful interdisciplinary research.

While controversies arise in the investigation of different approaches to language within the generative enterprise, it is difficult to reduce them to terminological issues. Linguistics is, of course, much more than a repertoire of terms and their proper use. As part of the generative enterprise, biolinguistics is a research program aiming to provide a genuine explanation for I-language. In order to engage in fruitful interdisciplinary work, it might be useful to bring to the fore the latest achievements in explaining I-language and why they are important, as well as the new problems and questions that they prompt.

\section{Anna Maria Di Sciullo}

\section{Evelina Leivada replies:}

Anna Maria Di Sciullo raises various interesting points. The main message of her response is that the use of the terms I identified and discussed as inaccurate is nowadays obsolete. This is not a matter on which we can agree to disagree, because it is not a matter of subjective opinion, but rather of checking the facts. As I suggested in my essay, the terms I discussed are not some barely encountered notions in present-day linguistics. Demonstrating this amounts to an inexpensive experiment that anyone can repeat at home. I should highlight that in doing this exercise in the present reply, I completely agree with Di Sciullo, who argues that we need to differentiate the various uses of the terms at different points in the development of the genera- 
tive enterprise. However, I insist that the issues discussed in my essay largely reflect the current lay of the land in mainly generative linguistics.

Consider the notion of a parameter, its various conceptions and its various sizes. Is this an obsolete discussion nowadays? If parameters have been reduced to a minimum, as Di Sciullo suggests in her letter, how many are they exactly, according to current linguistic theorizing? How many features? How many functional heads? This inability to provide even approximate numbers is very surprising, given Di Sciullo's claim that we have now successfully settled on the minimum numbers for each inventory. Even if, for the sake of discussion, we agree that we have reduced these inventories to the absolute minimum, have we reached consensus about whether this minimum number of parameters should be ascribed to the functional lexicon, to Universal Grammar, to externalization systems, or to third factor principles? Juxtapose Di Sciullo's view that parameters have been reduced to a minimum or subsumed in third factor principles with Luigi Rizzi's view that

the size of the set of parameters may well be large: cartographic studies suggest that the functional lexicon is very rich, hence if the parametrization is associated to this component the system will specify many parameters. ${ }^{30}$

Crucially, this claim was made in 2017, not 40 years ago. This serves to show that the ambiguity I identified regarding the use of certain terms refers to their current use, and is not obsolete as Di Sciullo suggests.

On May 7, 2020, three days after the publication of my essay in Inference-and 52 years and five days after the student revolution began in France-the first live debate from a series of video conferences called "Linguistics Flash-Mobs. Epic Battles in History" took place. ${ }^{31}$ In each conference, two seminal scholars were invited to discuss longstanding theoretical issues in linguistics. The first debate, moderated by Maria Rita Manzini and organized by Cecilia Poletto, hosted Giuseppe Longobardi and Ian Roberts, two distinguished professors of linguistics from York and Cambridge, respectively. The three questions that the two invitees addressed had to do with different aspects of one notion: parameters. In that debate, the discussion of parametric hierarchies made amply clear that for some linguists, parameters come in different sizes: macro, meso, and micro. This is part of the terminological fluidity that I analyzed in my essay and that Di Sciullo argues does not characterize the current state of affairs in the generative enterprise. This conference is not the exception. Parameters are very much part of the current lay of the land in generative linguistics and so is the quest to examine more structures from different languages and language families in order to uncover more of them, contra to Di Sciullo's claim that the field has now settled on the minimum number. ${ }^{32}$
Consider the usage of another term: linguistic genotype or genetic endowment for language. It has been suggested that "children have triggering experiences that stimulate their genetic properties to develop into their phenotypic properties [emphasis added]," ${ }^{33}$ that "linguistic knowledge is part of the child's genetic makeup [emphasis added], ${ }^{34}$ and that parameters are principles to which "you genetically fix the value [emphasis added]." ${ }^{35}$ These views, and many similar ones that are abundant in linguistics, imply the position that a part of the human genome is dedicated to language. Di Sciullo may have hoped that these claims belong to a different era and are no longer entertained. This is not the case. They are still popular, and mainstream enough to be diffused to the general public as established theses. A good example of how this is so is offered by Martin Haspelmath on his blog. ${ }^{36} \mathrm{He}$ presents part of the interview that linguist Jessica Coon gave on the occasion of the release of the 2016 movie Arrival, for which she did consulting work. The question was, "So if universal language theory only applies to humans, there's a real danger that if an alien race started communicating we'd have no hope of deciphering it?" Coon's reply was,

Yeah, definitely. When people talk about universal grammar it's just the genetic endowment that allows humans to acquire language. There are grammatical properties we could imagine that we just don't ever find in any human language, so we know what's specific to humans and our endowment for language. ${ }^{37}$

Perhaps an important question is whether there are more terms on which we should keep a close eye. In her letter, Di Sciullo correctly argues that it is helpful to consider the use of linguistic terms in neighboring sciences. She refers to a thesis she claims has been established: "Nonetheless, it has been established that Broca's area, corresponding to BA44/45 in the frontal lobe of the brain's dominant hemisphere, supports the processing of syntax in general." This looks like an oversimplified depiction of the neuroanatomical underpinnings of syntax. The way it is used in passing implies localization-a slippery terrain. Language operations do not reside in single brain regions; they are subserved by networks of brain regions. ${ }^{38}$ Certain operations used in syntax processing may typically have a certain distribution of labor, but the latter relies on networks spanning over many areas. In this sense, there is no one-to-one correspondence between a domain of linguistic analysis and an area in the brain.

To offer a second example, Di Sciullo uses the term "module," as I do too in my original essay, but this is yet another term that has been used in a terminologically fluid way. More specifically, Di Sciullo talks about modules of Universal Grammar, but she does not explain the sense in which the term is used. Again, this terminological imprecision is not without consequences for the field. It is not 
at all clear that Universal Grammar consists of modules or even that there is a language module per se. The term must be qualified, describing the sense in which it is employed; otherwise, its use may cause terminological confusion and erroneously exaggerate inexistent physical discreteness. ${ }^{39}$

There are several other points on which I respectfully disagree with Di Sciullo. For reasons of space I will discuss only two. First, she situates a biolinguistic approach to language within the generative enterprise: "Within the generative enterprise, the biolinguistic program is concerned with language internal to the individual, the I-language, which is distinct from the external language, the E-language." This is wrong, because biolinguistics is not specific to the generative approach. Of course, one can do excellent work on biolinguistics within a generative framework, but one can also do excellent work addressing questions about the evolution and biology of language, without using tools of generative linguistics. ${ }^{40}$ Second, Di Sciullo argues that even if terminological discrepancies exist, the crux of the matter in my essay is not terminology per se, but differing views of core concepts in linguistics. The answer to this is that I personally find it more useful to acknowledge the existence of a problem and then work on solving it, than to engage in chicken-or-egg dilemmas by discussing whether the focus should be on the cause versus the outcome, and on what counts as what. For me, the issue has to do with both the fluid way in which some terms are used to mean different things across studies (e.g., "parameters"), and with the use of terms that are simply wrong (e.g., "linguistic genotype"). It could be argued that the ambiguity that surrounds the use of some terms derives from different views of core concepts, but it could also be argued that some linguists hold alternative views of core concepts precisely because the terms have long been defined in ambiguous, unclear, or untenable ways. The question of what came first is not the most important one for me. The focus should not be on where ambiguity comes from, especially since we have reasonable knowledge about possible sources, and Di Sciullo also correctly identifies some, but on caring to acknowledge its existence and then work on it in a way that maximizes field-internal coherence and field-external visibility.

To conclude, I thank Anna Maria Di Sciullo for her response to my essay. I read it with great interest and I agree with the part of her epilogue that claims that "the terminological issues discussed in [Leivada's] essay should be understood as differences in the understanding of core concepts in linguistics." This is true; the contents of Di Sciullo's response made me realize once more that the use of many terms in linguistics is a matter of perspective, and a good degree of the terminological unclarity I talked about derives precisely from the holding of different perspectives. Terms like "the syntax area of the brain," "module," and "I- versus E-language" are not included in my original list of misused, ambiguous, and polysemous terms, so per- haps a follow-up study is due. With respect to the main point of Di Sciullo's critique, I want to reaffirm that the terms I discussed, together with their portrayed use, are not absent from present-day linguistics. I encourage readers to go through Di Sciullo's arguments carefully and then also read Juan Uriagereka's response to my original essay. I think Uriagereka is right about everything he writes in his letter. He is especially right about the title he chose to put on his reply. For some or perhaps even many linguists, these truly are terms of endearment. For this reason, attempts to discuss problems in their use may be brushed aside as not necessary, not timely, or not appropriate, especially if coming from junior scholars. But as the students in the French revolution of May 1968 said, if not us, who? If not now, when?

Anna Maria Di Sciullo is Director of the Interface Asymmetry Lab and Professor of Linguistics at the Université du Québec à Montréal.

\section{Evelina Leivada is a psycholinguist at the Universitat} Rovira $i$ Virgili in Tarragona, Spain.

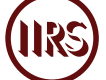

1. Noam Chomsky, "The Dewey Lectures 2013: What Kind of Creatures Are We? Lecture I: What Is Language?" The Journal of Philosophy 110, no. 12 (2013): 647, doi:10.5840/ jphil2013110121; Chomsky, Second Lecture of the UCLA Lectures (April 30, 2019).

2. Robert Berwick and Chomsky, "The Biolinguistic Program: The Current State of its Development," in The Biolinguistic Enterprise, ed. Anna Maria Di Sciullo and Cedric Boeckx (Oxford: Oxford University Press, 2011), 19-41; Anna Maria Di Sciullo and Lyle Jenkins, "Biolinguistics and the Human Language Faculty," Language 92, no. 3 (2017): el-e32, doi:10.1353/lan.2016.0056.

3. Noam Chomsky, "On the Evolution of Language: A Biolinguistic Perspective," an interview by Chronis Polychroniou, September 24, 2016; Berwick and Chomsky, Why Only Us: Language and Evolution (Cambridge: MIT Press, 2016); Noam Chomsky, "The Language Capacity: Architecture and Evolution," Psychonomic Bulletin and Review 24, no. 1 (2017): 2,000-2,003, doi:10.3758/s13423-016-1078-6; Johan Bolhuis et al., "How Could Language Have Evolved?” PLOS Biology 12, no. 8 (2014), doi:10.1371/journal.pbio.1001934.

4. Noam Chomsky, Language and Mind (New York: Harcourt Brace Jovanovich, Inc., 1973); Noam Chomsky, "Review of Skinner's Verbal Behavior," Language 35 (1959): 26-58; Eric Lenneberg, “On Explaining Language," Science, New Series 164, no. 3,880 (1969): 642.

5. Noam Chomsky, Syntactic Structures (The Hague: Mouton, 1957); Noam Chomsky, Aspects of the Theory of Syntax 
(Cambridge: MIT Press, 1965); Noam Chomsky, "Remarks on Nominalization," in Readings in English Transformational Grammar, ed. Roderick Jacobs and Peter Rosenbaum (Waltham: Ginn, 1970), 184-221; Noam Chomsky, Lectures on Government and Binding: The Pisa Lectures (Dordrecht: Foris, 1981); Noam Chomsky, The Minimalist Program (Cambridge: MIT Press, 1995); Noam Chomsky, "Problems of Projection," Lingua 130 (2013): 33-49, doi:10.1016/j. lingua.2012.12.003; Noam Chomsky, Ángel Gallego, and Dennis Ott, "Generative Grammar and the Faculty of Language: Insights, Questions, and Challenges," Catalan Journal of Linguistics (2019): 229-61, doi:10.5565/rev/catjl.288.

6. NoamChomsky, “ThreeFactorsinLanguage Design,” Linguistic Inquiry 36 (2005): 1-22, doi:10.1162/0024389052993655.

7. David Poeppel, "The Maps Problem and the Mapping Problem: Two Challenges for a Cognitive Neuroscience of Speech and Language," Cognitive Neuropsychology 29 (2012): 34-55, doi:10.1080/02643294.2012.710600.

8. Michiru Makuuchi et al., "Segregating the Core Computational Faculty of Human Language from Working Memory," Proceedings of the National Academy of Sciences 106 (2009): 8,362-67, doi:10.1073/pnas.0810928106; Yosef Grodzinsky and Andrea Santi, "Working Memory and Syntax Interact in Broca's Area," NeuroImage 37 (2007): 8-17, doi:10.1016/j. neuroimage.2007.04.047; Yosef Grodzinsky and Andrea Santi, "fMRI Adaptation Dissociates Syntactic Complexity Dimensions," NeuroImage 51 (2010): 1,285-93, doi:10.1016/j. neuroimage.2010.03.034.

9. Yosef Grodzinsky and Angela Friederici, "Neuroimaging of Syntax and Syntactic Processing," Current Opinion in Neurobiology 16 (2006): 240-46, doi:10.1016/j.conb.2006.03.007; Makuuchi et al., "Segregating the Core Computational Faculty"; Santi and Grodzinksy, "fMRI Adaptation"; Andrea Moro et al., "Syntax and the Brain: Disentangling Grammar by Selective Anomalies," NeuroImage 13 (2001): 110-18, doi:10.1006/nimg.2000.0668; Angela Friederici et al., "Language, Mind and Brain," Nature Human Behaviour 1 (2017): 713-22, doi:10.1038/s41562-017-0184-4.

10. Chomsky, Lectures on Government and Binding; Chomsky, Aspects of the Theory of Syntax.

11. Joseph Greenberg, "Some Universals of Grammar with Particular Reference to the Order of Meaningful Elements," in Universals of Language, ed. Greenberg (Cambridge: MA, MIT Press, 1963), 40-70.

12. Guglielmo Cinque, "Deriving Greenberg's Universal 20 and Its Exceptions," Linguistic Inquiry 36, no. 3 (2006): 315-32, doi:10.1162/0024389054396917.

13. Anna Maria Di Sciullo and Marco Nicolis, "Third Factor in the Development of P," paper delivered at the North East Linguistic Society 42, University of Toronto (2011); Anna Maria Di Sciullo, Marco Nicolis, and Stanca Somesfalean, "Comitative $P$," in Oxford Studies in Comparative Syntax: Variation in Prepositions, Comparative Approaches to Adpositional Phrases, ed. Jacopo Garzonio and Silvia Rossi (Oxford: Oxford University Press, 2020), 218-44.
14. Chomsky, The Minimalist Program; Noam Chomsky, "Minimalist Inquiries: The Framework," in Step by Step, ed. Roger Martin et al. (Cambridge: MIT Press, 2000), 89-155.

15. Christopher Collins, "Home Sweet Home," in NYU Working Papers in Linguistics 1 (2007): 1-34.

16. Chomsky, Reflections on Language (New York: Pantheon, 1975): 119.

17. Luigi Rizzi, Issues in Italian Syntax (Dordrecht: Foris, 1982); Hagit Borer, Parametric Syntax (Dordrecht: Foris Publications, 1984); Mark Baker, The Polysynthesis Parameter (New York: Oxford University Press, 1996); Ur Shlonsky, "The Cartographic Enterprise in Syntax," Language and Linguistic Compass 4, no. 6 (2010): 417-29, doi:10.1111/j.1749818X.2010.00202.x; Luigi Rizzi and Guglielmo Cinque, "Functional Categories and Syntactic Theory," Annual Review of Linguistics 2, no. 1 (2016): 139-63, doi:10.1146/ annurev-linguistics-011415-040827; Richard Kayne, Questions of Syntax (Oxford: Oxford University Press, 2019); Ian Roberts, Parameter Hierarchies and Universal Grammar (Oxford: Oxford University Press, 2019).

18. Baker, The Polysynthesis Parameter.

19. Richard Kayne, "Why There Are No Directionality Parameters," in Proceedings of the 28th West Coast Conference on Formal Linguistics, ed. Mary Byram Washburn et al. (Somerville, MA: Cascadilla Proceedings Project, 2011), 1-23.

20. Roberts, Parameter Hierarchies and Universal Grammar.

21. Chomsky, Lectures on Government and Binding.

22. Noam Chomsky, Knowledge of Language: Its Nature, Origin, and Use (New York: Praeger, 1986); Noam Chomsky, "Poverty of Stimulus: Unfinished Business," Studies in Chinese Linguistics 33, no. 1 (2012): 3-16.

23. Chomsky, Aspects of the Theory of Syntax.

24. Chomsky, "Remarks on Nominalization."

25. For example, according to case theory, postulated in the principles and parameters model, all nominal constituents are case marked, whether the case markers are overt, e.g., in Latin, old Germanic, or not, e.g., in Italian, English. Only [-N] categories, namely verbs and prepositions, assign case to their nominal complement. An empirical consequence of case theory is that in languages such as Italian and English, a preposition intervened between the first and the second nominal constituent, e.g., the demonstration of the theorem vs. *the demonstration the theorem. In the minimalist program, case theory has been eliminated; along with the other principles of UG postulated previously.

26. Chomsky, The Minimalist Program; David Adger, Core Syntax: A Minimalist Approach (Cambridge: MIT Press, 2003); David Pesetsky and Ester Torrego, "The Syntax of Valuation and Interpretability of Features," in Phrasal and Clausal Architecture: Syntactic Derivation and Interpretation, ed. Simin Karimi, Vida Samiian, and Wendy Wilkins (Amsterdam: John Benjamins, 2004), 262-93; Anna Maria Di Sciullo and Daniela Isac, "The Asymmetry of Merge," Biolinguistics 2: (2008): 260-90.

27. Chomsky, "Problems of Projection"; Noam Chomsky, "Prob- 
lems of Projection: Extensions," in Structures, Strategies and Beyond: Studies in Honour of Adriana Belletti, ed. Elisa Di Domenico et al. (Amsterdam: John Benjamins, 2015), 3-16; Chomsky, Gallego, and Ott, "Generative Grammar and the Faculty of Language."

28. Marc Hauser, Noam Chomsky, and W. Tecumseh Fitch, "The Faculty of Language: What Is It, Who Has It, and How Did It Evolve?" Science 298 (2002): 1,569-79, doi:10.1126/science.298.5598.1569.

29. Chomsky, UCLA Lectures (2019).

30. Luigi Rizzi, "On the Format and Locus of Parameters: The Role of Morphosyntactic Features," Linguistic Analysis 41, no. 3 (2017): 159-91.

31. "Linguistics Flash-Mobs. Epic Battles in History," YouTube channel.

32. On the issue of parametrization, see, for instance, Sandra Quarezemin, "Cartography, Left Periphery and Criterial Positions: An Interview with Luigi Rizzi," DELTA: Documentação de Estudos em Lingüística Teórica e Aplicada 36, no. 1 (2020), doi:10.1590/1678-460x2020360110.

33. David Lightfoot, How New Languages Emerge (Cambridge: Cambridge University Press. 2006), 46.

34. Rosalind Thornton and Ken Wexler, Principle B, VP Ellipsis, and Interpretation in Child Grammar (Cambridge: MIT Press, 1999), 1.
35. Noam Chomsky, "Conclusion," in Of Minds and Language: A Dialogue with Noam Chomsky in the Basque Country, ed. Massimo Piattelli-Palmarini, Pello Salaburu, and Juan Uriagereka (Oxford: Oxford University Press, 2009), 385.

36. Martin Haspelmath, "Chomsky Now Rejects Universal Grammar (and Comments on Alien Languages)," Diversity Linguistics Comment (blog), September 13, 2018.

37. The interview was titled "The Real-Life Linguist behind Arrival Weighs the Chilling Challenges of Alien Contact," and it was given to Playboy in 2016. To the best of my knowledge, it is no longer available online. The part I provide here was taken from Martin Haspelmath's blogpost referenced above.

38. Peter Hagoort, "MUC (Memory, Unification, Control) and Beyond," Frontiers in Psychology 4 (2013): 416, doi:10.3389/ fpsyg.2013.00416.

39. See the limitations of the term "module" and a discussion of how organisms may develop modular behavior without consisting of modules in Mary Jane West-Eberhard, Developmental Plasticity and Evolution (Oxford: Oxford University Press, 2003), 81.

40. For a recent example of the latter, see Bart de Boer et al., "Evolutionary Dynamics Do Not Motivate a Single-Mutant Theory of Human Language," Scientific Reports 10, no. 1 (2020): 1-9, doi:10.1038/s41598-019-57235-8. 